\title{
Development of Simple Methods to Reduce the Exposure of the Public to Radiation from Patients Who Have Undergone 18F-FDG PET/CT
}

\author{
Razi Muzaffar ${ }^{1}$, Elyse Koester ${ }^{2}$, Sarah Frye ${ }^{3}$, Saud Alenezi ${ }^{1}$, Barbara B. Sterkel ${ }^{4}$, and Medhat M. Osman ${ }^{1}$ \\ ${ }^{1}$ Division of Nuclear Medicine, Department of Radiology, Saint Louis University, Saint Louis, Missouri; ${ }^{2}$ Division of Nuclear \\ Medicine, St. Louis University Hospital, SSM Health, St. Louis, Missouri; ${ }^{3}$ Medical Imaging and Radiation Therapeutics, Saint Louis \\ University, St. Louis, Missouri; and ${ }^{4}$ Diagnostic Imaging Service VA, St. Louis Health Care System, Saint Louis, Missouri
}

\begin{abstract}
At a time when reducing the radiation dose to patients and the public has become a major focus, we assessed the radiation exposure rate from patients after an ${ }^{18} \mathrm{~F}-\mathrm{FDG}$ PET/CT scan and evaluated different interventions to reduce it. Methods: We enrolled 100 patients, divided into 2 groups. For both groups, the radiation dose rate was measured with an ionization survey meter immediately after the scan. For group 1, the patients then voided and their dose rate was measured again. For group 2, the patients waited 30 min before voiding, and we measured the dose rate before (group 2A) and after (group 2B) they voided. Results: In total, 74 of the 100 patients exceeded the $20 \mu \mathrm{Sv} / \mathrm{h}$ ( $2 \mathrm{mR} / \mathrm{h}$ ) threshold immediately after the scan. In group 1 , the mean dose rate decreased by $20.0 \%$ from the postscan measurement, with 12 of 36 remaining at or above $20 \mu \mathrm{Sv} / \mathrm{h}$. In group $2 \mathrm{~A}$, the mean dose rate decreased by $23 \%$ from the postscan measurement, with 9 of 38 remaining at or above 20 $\mu \mathrm{Sv} / \mathrm{h}$. In group $2 \mathrm{~B}$, the mean dose rate decreased by $35 \%$ from the postscan measurement, with 1 of 38 remaining at $20 \mu \mathrm{Sv} / \mathrm{h}$. Conclusion: Nearly $75 \%$ of patients undergoing an ${ }^{18} \mathrm{~F}-\mathrm{FDG}$ PET/CT scan exceed $20 \mu \mathrm{Sv} / \mathrm{h}$ when leaving the imaging facility. The most effective method to reduce radiation exposure was to have the patient void 30 min after the examination.
\end{abstract}

Key Words: ALARA; PET/CT; radiation exposure; radiation safety

J Nucl Med Technol 2020; 48:63-67

DOI: 10.2967/jnmt.119.233296

$\mathbf{T}$ he increased use of diagnostic imaging throughout the world has caused a drastic increase in the radiation exposure of the population and has raised concerns about potential cancer risks associated with this trend, as well as unfavorable media coverage $(1,2)$. PET/CT has emerged as the gold standard to stage and restage various types of malignancies, while also seeing a steady incline in the number of studies performed.

\footnotetext{
Received Jul. 2, 2019; revision accepted Aug. 21, 2019.

For correspondence or reprints contact: Medhat M. Osman, Division of Nuclear Medicine, Department of Radiology, Saint Louis University, 3635 Vista Ave., 2-DT, St. Louis, MO 63110.

E-mail: medhat.osman@health.slu.edu

Published online Oct. 11, 2019.

COPYRIGHT (C) 2020 by the Society of Nuclear Medicine and Molecular Imaging.
}

These patients are receiving not only radiation from the CT component of the examination but also lingering radiation from the radiopharmaceutical, ${ }^{18} \mathrm{~F}-\mathrm{FDG}$. Compared with $\mathrm{CT}$ and radiography, both nuclear medicine and PET/CT studies inherently expose the general public to additional radiation due to the administration of radiopharmaceuticals. This concern has led to various new techniques and has paved the way for technologic advancements to both improve image quality and simultaneously reduce the radiation to which a patient is exposed.

The Nuclear Regulatory Commission has well-established guidelines for the release of patients undergoing therapeutic procedures (3). The maximum accepted exposure of the public from a material source-20 $\mu \mathrm{Sv} / \mathrm{h}(2 \mathrm{mR} / \mathrm{h})$-is used as a benchmark to reduce exposure to as low as reasonably achievable (4). However, guidelines addressing the release of a patient undergoing diagnostic nuclear medicine or PET/CT examinations are not as clear. Nevertheless, medical facilities are under increased scrutiny to reduce the radiation exposure of both patients and the public.

Although the half-life of ${ }^{18} \mathrm{~F}-\mathrm{FDG}$ is relatively short (110 min), many patients have multiple tests and clinical appointments in a single visit. It is therefore important to consider the time frame immediately after a scan. At a time when reducing the radiation dose to patients and imaging staff has become a major focus, this study set out to explore the effectiveness of reducing the radiation exposure of the public from a patient who received a standard-of-care ${ }^{18} \mathrm{~F}$-FDG PET/CT examination. We sought a low-cost solution with the least impact or inconvenience to the patient and the PET/CT department. In addition, renal function was evaluated as a potential variable affecting this exposure. As proof of principle, we also evaluated the difference in radiation exposure between a small group of patients undergoing ${ }^{18} \mathrm{~F}$-fluciclovine $\mathrm{PET} / \mathrm{CT}$, with an imaging time of 3-5 min after injection, and patients undergoing ${ }^{18} \mathrm{~F}$ FDG PET/CT, with an imaging time of $60 \mathrm{~min}$.

\section{MATERIALS AND METHODS}

This project was undertaken as a quality improvement initiative. The members of the Institutional Review Board reviewed this study and determined it did not need approval under their guidelines. 
Patients undergoing standard-of-care ${ }^{18}$ F-FDG PET/CT examinations who were willing to be included in our measurements were enrolled in the study until the total reached 100 patients $(61$ men and 39 women; mean age, $54 \mathrm{y}$ ). The patients received a weightbased ${ }^{18}$ F-FDG dose (range, 233.1-558.7 MBq [6.3-15.1 mCi]; mean, $421.8 \mathrm{MBq}$ [11.4 mCi]), which was followed by an approximately 60-min uptake time (range, 51-93 min; mean, $62 \mathrm{~min}$ ). They were then instructed to void immediately before a standardof-care whole-body PET/CT acquisition (acquisition time range, 14-47 min; mean, $26 \mathrm{~min}$ ). After the scan, each patient was then measured with an ionization survey meter (Victoreen ion chamber survey meter, model 450; Cardinal Health, Inc.) placed $1 \mathrm{~m}$ from the patient. A dedicated area within the hospital was used for measurement to ensure consistency. All measurements were performed by the same technologist, with the survey meter aimed at the bladder. Patients were randomly divided into 2 groups of 50 patients each based on availability after the PET/CT examination. For both groups, the radiation dose rate was measured with an ionization survey meter immediately after the scan. For group 1, the patients then voided and their dose rate was measured again. For group 2, the patients waited $30 \mathrm{~min}$ before voiding, and the dose rate was measured before (group 2A) and after (group 2B) they voided. Consideration of renal function was also investigated; renal function data and estimated glomerular filtration rate values within 2 wk of the scan were available for 77 of the 100 patients.

A small sample of 12 men (mean age, $62 \mathrm{y}$ ) undergoing standardof-care ${ }^{18} \mathrm{~F}$-fluciclovine PET/CT for biochemically recurrent prostate cancer was also evaluated. Although the ${ }^{18} \mathrm{~F}$ dose was comparable, the difference in protocols between the ${ }^{18} \mathrm{~F}$-fluciclovine and ${ }^{18} \mathrm{~F}$ FDG patients was assessed (3-5 vs. $60 \mathrm{~min}$ ). All patients received the standard 370-MBq (10-mCi) dose, and images were acquired 3-5 min afterward. On completion of imaging, each patient was measured with the ionization survey meter in the same way as for the ${ }^{18}$ F-FDG PET/CT scans. Patients were instructed to wait $30 \mathrm{~min}$ and dose rates were remeasured. Each patient was then instructed to void, and the final dose rates were measured.

\section{RESULTS}

In total, 74 of the 100 patients (74\%) exceeded $20 \mu \mathrm{Sv} / \mathrm{h}$ $(2 \mathrm{mR} / \mathrm{h})$ at $1 \mathrm{~m}$ immediately after their PET/CT scan. Of these, $36(49 \%)$ were from group 1 and $38(51 \%)$ were from

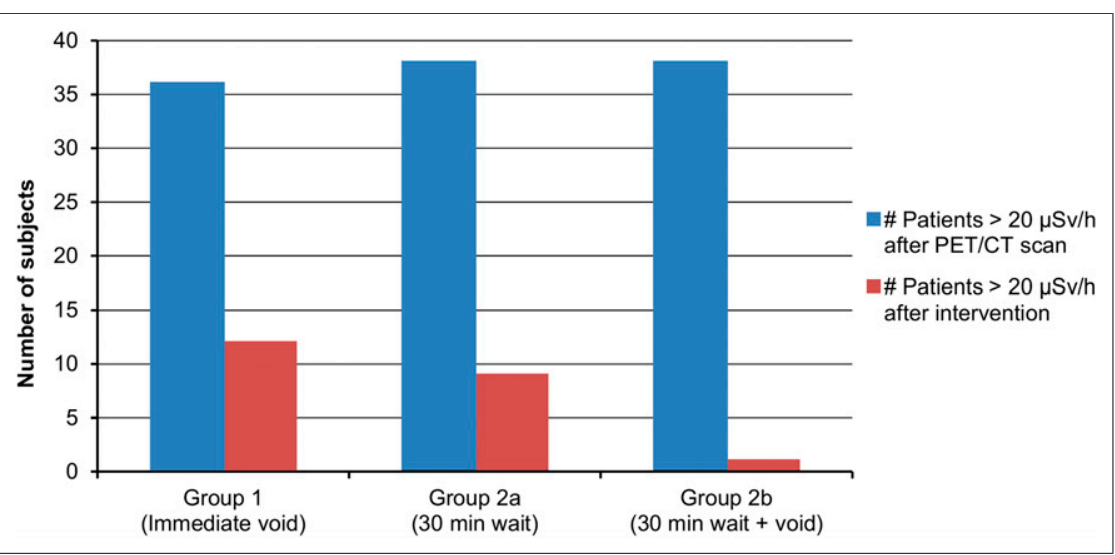

FIGURE 1. Comparison of patients above $20 \mu \mathrm{Sv} / \mathrm{h}$ threshold measured immediately after PET/CT scan vs. patients above $20 \mu \mathrm{Sv} / \mathrm{h}$ after various interventions to reduce radiation exposure. groups $2 \mathrm{~A}$ and $2 \mathrm{~B}$. In group 1, the mean dose rate decreased by $20.0 \%$ (from 23 to $18.3 \mu \mathrm{Sv} / \mathrm{h}$ ) from the postscan measurement, with 12 of $36(33 \%)$ remaining at or above the 20 $\mu \mathrm{Sv} / \mathrm{h}$ threshold. In group $2 \mathrm{~A}$, the mean dose rate decreased by $23 \%$ (from 23.4 to $18 \mu \mathrm{Sv} / \mathrm{h}$ ) from the postscan measurement, with 9 of $38(24 \%)$ remaining at or above $20 \mu \mathrm{Sv} / \mathrm{h}$. In group 2B, the mean dose rate decreased by $35 \%$ (from 23.4 to $15.1 \mu \mathrm{Sv} / \mathrm{h}$ ) from the postscan measurement, with 1 of 38 (3\%) remaining at $20 \mu \mathrm{Sv} / \mathrm{h}$ (Fig. 1).

Of the 77 patients with renal function data, only $12(16 \%)$ had reduced renal function (estimated glomerular filtration rate $<60)$. Of these, $11(92 \%)$ were above $20 \mu \mathrm{Sv} / \mathrm{h}$ immediately after the scan, whereas $47(72 \%)$ of the 65 patients with normal estimated glomerular filtration rate were above $20 \mu \mathrm{Sv} / \mathrm{h}$ after the scan (Fig. 2). The data suggest that patients with reduced renal function have higher radiation exposure than those with normal renal function. However, this difference was not statistically significant, with a $P$ value of 0.274 .

Of the 12 patients undergoing ${ }^{18} \mathrm{~F}$-fluciclovine $\mathrm{PET} / \mathrm{CT}$, $12(100 \%)$ remained at or above $20 \mu \mathrm{Sv} / \mathrm{h}$ immediately after the scan. After a 30-min wait, the mean exposure decreased by $25 \%$ (from 28.2 to $21.2 \mu \mathrm{Sv} / \mathrm{h}$ ), but 9 patients (75\%) remained at or above $20 \mu \mathrm{Sv} / \mathrm{h}$. After a 30-min wait followed by voiding, the mean exposure decreased by $30 \%$ (from 28.2 to $19.8 \mu \mathrm{Sv} / \mathrm{h}$ ), but 9 patients $(75 \%)$ remained at or above $20 \mu \mathrm{Sv} / \mathrm{h}$. When comparing the most effective intervention-waiting $30 \mathrm{~min}$ plus voiding-between the ${ }^{18} \mathrm{~F}-\mathrm{FDG}$ and ${ }^{18} \mathrm{~F}$-fluciclovine studies, we found that $97 \%$ (37/38) of the ${ }^{18} \mathrm{~F}-\mathrm{FDG}$ patients who initially exceeded 20 $\mu \mathrm{Sv} / \mathrm{h}$ fell below that threshold, compared with only $25 \%$ $(3 / 12)$ of the ${ }^{18} \mathrm{~F}$-fluciclovine patients (Fig. 3).

\section{DISCUSSION}

The ever-expanding use of diagnostic imaging continues to raise concerns about the increased cancer risk from ionizing radiation exposure. This issue has been especially critical for children and young adults. Multiple media reports have surfaced describing the increased cancer risk from medical imaging examinations. During this same period, the use of diagnostic imaging has been increasing at a steady pace. Although the natural background radiation has not significantly changed, the radiation exposure from medical imaging has increased more than 6-fold. According to a report issued in March 2009 by the National Council on Radiation Protection and Measurements, medical imaging contributed about $15 \%$ of the overall radiation dose in the United States in the 1980s, compared with about 50\% in 2006 (5). The use of PET/CT in the clinical setting has also been expanding 


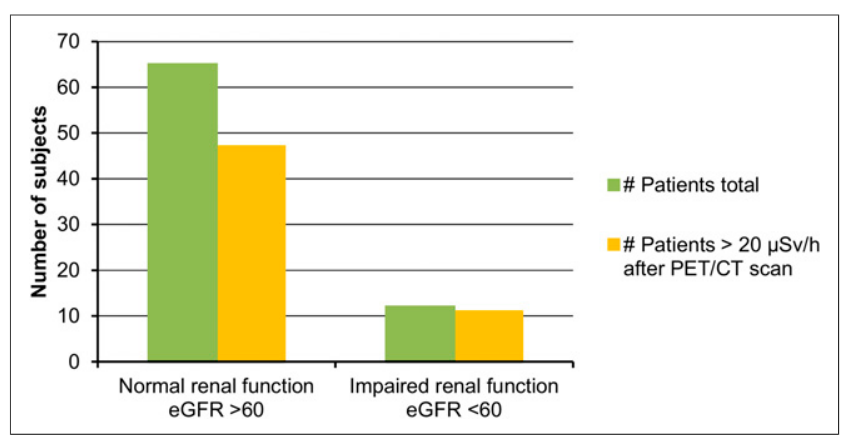

FIGURE 2. Comparison of patients with normal estimated glomerular filtration rate (eGFR) vs. patients with abnormal eGFR. Shown are total patients in each group based on eGFR, as well as patients above $20 \mu \mathrm{Sv} / \mathrm{h}$ threshold immediately after imaging.

with the advancement of medicine and technology. The number of PET/CT systems increased over 10-fold since 2001, and the number of clinical scans performed in the United States alone is estimated at nearly 2 million, which is an increase of $13 \%$ over $2015(6,7)$.

The concerns about exposure of the public to ionizing radiation from medical imaging have brought about a movement in both the pediatric population with the Image Gently campaign and the adult population with the Image Wisely campaign. To maximize the risk-to-benefit ratio, the medical community attempts to optimize diagnostic image quality while at the same time following the as-low-asreasonably-achievable principle. The Image Gently and Image Wisely campaigns were developed to educate both providers and consumers in an attempt to curtail unnecessary imaging and reduce radiation exposure $(8-10)$. However, there continues to be debate on whether low-dose radiation exposure can cause cancer (11). In a recent publication, Siegel et al. made the case for terminating these campaigns because of the erroneous extrapolation of the linear no-threshold model from high- to low-dose radiation as well as propagation of radiophobia. The linear no-threshold

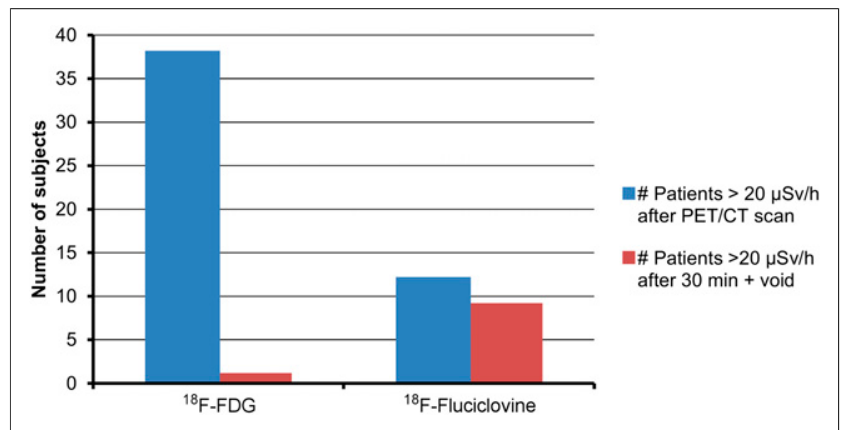

FIGURE 3. Comparison of radiation exposure from patients undergoing ${ }^{18} \mathrm{~F}-\mathrm{FDG} \mathrm{PET} / \mathrm{CT}$ scan vs. ${ }^{18} \mathrm{~F}$-fluciclovine PET/CT scan. Shown are number of patients exceeding $20 \mu \mathrm{Sv} / \mathrm{h}$ threshold immediately after scan, as well as number of patients continuing to exceed $20 \mu \mathrm{Sv} / \mathrm{h}$ after waiting $30 \mathrm{~min}$ and then voiding. model for radiation-induced cancer has guided radiation protection policies since the 1950s and is supported by national and international advisory bodies. It is based on the concept that low levels of radiation increase mutations, which lead to increased cancers. However, Siegel et al. claim that low-dose radiation may even help prevent cancer by inducing repair of preexisting and ongoing DNA damage while repairing radiogenic damage (12-15). Nevertheless, from the regulatory standpoint, the linear no-threshold model is accepted and continues to be supported by national advisory bodies (National Research Council and National Council on Radiation Protection and Measurements) $(16,17)$. Furthermore, those questioning the linear no-threshold model are basing the risk versus benefit on only the patient. This point of view should not be the same for the general public or potentially vulnerable patient populations in hospital or clinic waiting areas. It has been shown that the cancer risk increases substantially in patients with a suppressed immune system such as in young AIDS patients, for whom the cancer incidence increases by a factor of 40 , or in young organ-transplant patients, for whom the cancer mortality rate increases by a factor of $60(18,19)$. Nonetheless, a balance needs to be made between radiation risk and radiation aversion.

Various techniques have been developed to decrease the level of radiation exposure by using weight-based protocols for both the radiopharmaceutical delivered and the CT scan. Furthermore, PET/CT systems with higher sensitivity and improved performance can take advantage of enhanced detector technology (better time-of-flight performance, continuous bed motion, or an extended axial field of view) to reduce the ${ }^{18}$ F-FDG dose $(20,21)$. According to the Society of Nuclear Medicine and Molecular Imaging guidelines, the typical administered ${ }^{18} \mathrm{~F}-\mathrm{FDG}$ dose is $370-740 \mathrm{MBq}(10-20 \mathrm{mCi})$ (22). However, patients who weigh more than $75 \mathrm{~kg}$ should receive a slightly higher dose to compensate for degraded image quality due to a lower signal-to-noise ratio from excessive attenuation (20).

Although these advancements and techniques address the radiation exposure of patients and radiation workers, they do not address the exposure of the public to patients or the repeated exposure of unmonitored nonradiologic medical staff to patients. Many patients schedule multiple tests and clinical appointments on a single day, exposing not only other patients in waiting areas but also general medical personnel. Multiple studies have been performed to assess the amount of radiation received by medical personnel in various roles (23). Regardless of the validity of the linear no-threshold model, there is justification for the radiation exposure of both patients and radiation workers; however, the same cannot be said for the general public.

Our study evaluated simple noninvasive interventions to reduce the overall radiation exposure of the public with no additional cost and only an additional 30-min wait for the patient before being released after imaging. At a time when the Nuclear Regulatory Commission has strict guidelines 
on the release of a patient treated with a radioisotope, the recommendations about releasing a patient undergoing diagnostic PET/CT are not as clear. In addition, national and international guidelines (Society of Nuclear Medicine and Molecular Imaging, American College of Radiology, and European Association of Nuclear Medicine) neither give recommendations for radiation exposure from patients nor recommend voiding or waiting after a scan before release $(20,22,24)$. Although our data on the reduced radiation exposure after voiding were similar to prior studies, we found that nearly $75 \%$ of patients undergoing an ${ }^{18} \mathrm{~F}-\mathrm{FDG}$ PET/CT scan leave the imaging facility exceeding $20 \mu \mathrm{Sv} / \mathrm{h}$ at $1 \mathrm{~m}(25)$. We also attempted to address the concern about reduced renal function in these patients. Although renal failure has been shown not to have a significant impact on ${ }^{18}$ F-FDG biodistribution in PET/CT, our initial data showed a possible correlation between impaired renal function and elevated radiation exposure (26). However, because of our small sample size, this correlation was not statistically significant.

Moreover, the initial data from patients undergoing a ${ }^{18} \mathrm{~F}$ fluciclovine study showed that they exposed the public to a higher level of radiation than did patients undergoing an ${ }^{18}$ F-FDG study. The higher exposure is most likely due to the elimination of the 60-min uptake phase, given the different pharmacodynamics. Sörensen et al. demonstrated that tumor uptake of ${ }^{18} \mathrm{~F}$-fluciclovine peaks at around $3 \mathrm{~min}$, with a plateau from 3 to 12 min and gradual washout. Lymph node uptake was rapid, with washout even faster than from tumor, and the urinary bladder had gradual uptake (27). Therefore, standard imaging with ${ }^{18} \mathrm{~F}$-fluciclovine is performed 3-5 min after radiotracer administration, compared with $60 \mathrm{~min}$ with ${ }^{18} \mathrm{~F}-\mathrm{FDG}$ (28). Although the ${ }^{18} \mathrm{~F}$ isotope is the same in both the ${ }^{18} \mathrm{~F}$-fluciclovine and the ${ }^{18} \mathrm{~F}$-FDG studies, as well as there being a similar injected dose-370 versus $421.8 \mathrm{MBq}$ (10 vs. $11.4 \mathrm{mCi}$ ) - the difference in the protocol with ${ }^{18} \mathrm{~F}$-fluciclovine requires a significantly shorter uptake phase. In our study, this difference caused $75 \%$ of the ${ }^{18} \mathrm{~F}$-fluciclovine patients to remain above the $20 \mu \mathrm{Sv} / \mathrm{h}$ threshold after waiting $30 \mathrm{~min}$ plus voiding, versus only $3 \%$ for the ${ }^{18} \mathrm{~F}$-FDG patients.

This study may be the first to evaluate the radiation exposure of the public to a patient undergoing an ${ }^{18} \mathrm{~F}-\mathrm{FDG}$ PET/CT scan and to assess the feasibility of implementing simple interventions to significantly reduce that exposure. Other studies have addressed radiation exposure but in the context of exposure to a patient or technologist $(25,29)$. In addition, the effects of voiding after imaging to reduce exposure have also been studied, but not the combination of waiting plus voiding. In theory, these principles can also be used for other diagnostic PET and nuclear medicine studies before a patient leaves an imaging facility.

Our study is not without limitations. The relatively small sample size limits the statistical significance of the findings, especially when evaluating the effect of renal impairment. A larger sample size could allow for evaluation of various stages and severities of renal disease. Additionally, only 12 patients undergoing an ${ }^{18} \mathrm{~F}$-fluciclovine study were evaluated. Other commonly used Food and Drug Administrationapproved PET tracers such as ${ }^{18} \mathrm{~F}$-sodium fluoride and ${ }^{68}$ Ga-DOTATATE should also be evaluated. We found that even the same isotope can expose the public to vastly different levels of radiation, depending on the protocol. Lastly, this study was limited to the experience of a single institution with a fixed scanner. Because of financial concerns, a fixed PET/CT scanner is not always cost-effective and a mobile PET/CT scanner is often used. However, given the space and time constraints of a mobile scanner, these simple interventions may not be feasible. A prospective multiinstitute study would be better able to assess different patient populations, radiotracers, and protocols and to compare both fixed and mobile facilities.

\section{CONCLUSION}

Current guidelines do not advocate measuring the radiation level emanating from patients before they are released after undergoing ${ }^{18} \mathrm{~F}-\mathrm{FDG}$ PET/CT. Our analysis shows that this level exceeds the $20 \mu \mathrm{Sv} / \mathrm{h}$ threshold in nearly $75 \%$ of patients after ${ }^{18} \mathrm{~F}$-FDG PET/CT and in all patients after ${ }^{18} \mathrm{~F}$ flucicolvine PET/CT. For ${ }^{18} \mathrm{~F}-\mathrm{FDG}$, the most effective method to reduce this level was to have the patient wait $30 \mathrm{~min}$ after the examination and then void, with $97 \%$ of patients then being below the threshold. This simple intervention is especially important when patients have additional appointments and are in close contact with others. However, this intervention was not as effective for ${ }^{18} \mathrm{~F}$-fluciclovine, dropping only $25 \%$ of patients below the threshold. Therefore, more effective techniques should be developed and validated for non- ${ }^{18}$ F-FDG PET/CT scans.

\section{DISCLOSURE}

No potential conflict of interest relevant to this article was reported.

\section{REFERENCES}

1. Morin RL, Seibert JA, Boone JM. Radiation dose and safety: informatics standards and tools. J Am Coll Radiol. 2014;11(12 pt B):1286-1297.

2. Berrington de González A, Mahesh M, Kim K-P, et al. Projected cancer risks from computed tomographic scans performed in the United States in 2007. Arch Intern Med. 2009;169:2071-2077.

3. 35.75 Release of individuals containing unsealed byproduct material or implants containing byproduct material. U.S. Nuclear Regulatory Commission website. http://www.nrc.gov/reading-rm/doc-collections/cfr/part035/part035-0075.html. Updated August 29, 2017. Accessed November 13, 2019.

4. Howe DB, Beardsly M, Basksh S. Consolidated Guidance about Materials Licenses: Program-Specific Guidance about Medical Use Licenses Final Report (NUREG-1556. Vol. 9, Rev. 2). Washington, DC: U.S. Nuclear Regulatory Commission; 2008.

5. Bolus NE. NCRP report 160 and what it means for medical imaging and nuclear medicine. J Nucl Med Technol. 2013;41:255-260.

6. 2011 PET Imaging Market Summary Report. Greenbelt, MD: IMV; 2011.

7. PET Imaging Market Summary Report 2019. AuntMinnie website. IMV: PET/CT drives PET scan volume to new heights. https://www.auntminnie.com/index.aspx? $\mathrm{sec}=$ sup\&sub=mol\&pag=dis\&ItemID=125533.

8. Brink JA, Amis ES. Image Wisely: A Campaign to Increase Awareness about Adult Radiation Protection. Radiology. 2010;257:601-602.

9. Goske MJ, Applegate KE, Boylan J, et al. The Image Gently campaign: working together to change practice. AJR. $2008 \mathrm{Feb}$;190(2):273-4.m 
10. Cohen MD. ALARA, Image gently and CT-induced cancer. Pediatr Radiol. 2015;45:465-470.

11. Doss M. Are we approaching the end of the linear no-threshold era? J Nucl Med. 2018;59:1786-1793.

12. Siegel JA, Sacks B, Pennington CW, et al. Dose optimization to minimize radiation risk for children undergoing $\mathrm{CT}$ and nuclear medicine imaging is misguided and detrimental. J Nucl Med. 2017;58:865-868.

13. Siegel JA, Pennington CW, Sacks B. Subjecting radiologic imaging to the linear no-threshold hypothesis: a non sequitur of non-trivial proportion. J Nucl Med. 2017;58:1-6.

14. Duncan JR, Lieber MR, Adachi N, et al. DNA repair after exposure to ionizing radiation is not error-free [letter]. $J$ Nucl Med. 2018;59:348.

15. Siegel JA, Sacks B, Pennington CW, et al. DNA repair after exposure to ionizing radiation is not error-free [reply]. J Nucl Med. 2018;59:349.

16. National Research Council of the National Academies. Health Risks from Exposure to Low Levels of Ionizing Radiation: BEIR VII Phase 2. Washington, DC: The National Academies Press; 2006:424.

17. NCRP. Commentary No. 27: Implications of Recent Epidemiologic Studies for the Linear-Nonthreshold Model and Radiation Protection. Bethesda, MD: National Council on Radiation Protection and Measurements; 2018:210.

18. Oliveira Cobucci RN, Saconato H, Lima PH, et al. Comparative incidence of cancer in HIV-AIDS patients and transplant recipients. Cancer Epidemiol. 2012;36:e69-e73.

19. Biggar RJ, Frisch M, Goedert JJ; AIDS-Cancer Match Registry Study Group. Risk of cancer in children with AIDS. JAMA. 2000;284:205-209.

20. Boellaard R, Delgado-Bolton R, Oyen WJ, et al. FDG PET/CT: EANM procedure guidelines for tumour imaging: version 2.0. Eur J Nucl Med Mol Imaging. $2015 ; 42: 328-354$
21. Murray I, Kalemis A, Glennon J, et al. Time-of-flight PET/CT using low-activity protocols: potential implications for cancer therapy monitoring. Eur J Nucl Med Mol Imaging. 2010;37:1643-1653.

22. Jadvar H, Colletti PM, Delgado-Bolton R, et al. Appropriate use criteria for ${ }^{18} \mathrm{~F}$ FDG PET/CT in restaging and treatment response assessment of malignant disease. J Nucl Med. 2017;58:2026-2037.

23. Kitahara CM, Linet MS, Balter S, et al. Occupational radiation exposure and deaths from malignant intracranial neoplasms of the brain and CNS in U.S. radiologic technologists, 1983-2012. AJR. 2017;208:1278-1284.

24. ACR-SPR practice parameter for performing FDG-PET/CT in oncology. American College of Radiology website. https://www.acr.org/-/media/ACR/ Files/Practice-Parameters/fdg-pet-ct.pdf. Revised 2016. Accessed November $13,2019$.

25. Madsen MT, Anderson JA, Halama JR, et al. AAPM task group 108: PET and PET/CT shielding requirements. Med Phys. 2006;33:4-15.

26. Kode V, Karsch H, Osman MM, et al. Impact of renal failure on F-18-FDG PET/ CT scans. Front Oncol. 2017;7:155.

27. Sörensen J, Owenius R, Lax M, et al. Regional distribution and kinetics of $\left[{ }^{18} \mathrm{~F}\right]$ fluciclovine (anti- $\left[{ }^{18} \mathrm{~F}\right] \mathrm{FACBC}$ ), a tracer of amino acid transport, in subjects with primary prostate cancer. Eur J Nucl Med Mol Imaging. 2013;40: 394-402.

28. Axumin fluciclovine F18 injection, for intravenous use. https://www.axumin.com/ sites/default/files/2018-03/Axumin_PI_08_2016_Clean.pdf. Published 2016. Accessed November 13, 2019.

29. Bayram T, Yilmaz AH, Demir M, et al. Radiation dose to technologists per nuclear medicine examination and estimation of annual dose. J Nucl Med Technol. 2011; 39:55-59. 